\title{
Lattice-Boltzmann simulations of the drag force on a sphere approaching a superhydrophobic striped plane
}

\author{
Alexander L. Dubov, ${ }^{1,2}$ Sebastian Schmieschek, ${ }^{3}$ Evgeny S. \\ Asmolov, ${ }^{1,4,5}$ Jens Harting,,$^{3,6}$ and Olga I. Vinogradova ${ }^{1,2,7}$ \\ ${ }^{1}$ A.N. Frumkin Institute of Physical Chemistry and Electrochemistry, \\ Russian Academy of Sciences, 31 Leninsky Prospect, 119071 Moscow, Russia \\ ${ }^{2} D W I$ - Leibniz Institute for Interactive Materials, \\ RWTH Aachen, Forckenbeckstraße 50, 52056 Aachen, Germany \\ ${ }^{3}$ Department of Applied Physics, Eindhoven University of Technology, \\ P.O. Box 513, 5600 MB Eindhoven, The Netherlands \\ ${ }^{4}$ Central Aero-Hydrodynamic Institute, 140180 Zhukovsky, Moscow region, Russia \\ ${ }^{5}$ Institute of Mechanics, M.V. Lomonosov Moscow State University, 119991 Moscow, Russia \\ ${ }^{6}$ Institute for Computational Physics, University of Stuttgart, Allmandring 3, 70569 Stuttgart, Germany \\ ${ }^{7}$ Department of Physics, M.V. Lomonosov Moscow State University, 119991 Moscow, Russia
}

\begin{abstract}
By means of lattice-Boltzmann simulations the drag force on a sphere of radius $R$ approaching a superhydrophobic striped wall has been investigated as a function of arbitrary separation $h$. Superhydrophobic (perfect-slip vs. no-slip) stripes are characterized by a texture period $L$ and a fraction of the gas area $\phi$. For very large values of $h / R$ we recover the macroscopic formulae for a sphere moving towards a hydrophilic no-slip plane. For $h / R=O(1)$ and the drag force is smaller than predicted by classical theories for hydrophilic no-slip surfaces, but larger than expected for a sphere interacting with a uniform perfectly slipping wall. At a thinner gap, $h \ll R$ the force reduction compared to a classical result becomes more pronounced, and is maximized by increasing $\phi$. In the limit of very small separations our simulation data are in quantitative agreement with an asymptotic equation, which relates a correction to a force for superhydrophobic slip to texture parameters. In addition, we examine the flow and pressure field and observe their oscillatory character in the transverse direction in the vicinity of the wall, which reflects the influence of the heterogeneity and anisotropy of the striped texture. Finally, we investigate the lateral force on the sphere, which is detectable in case of very small separations and is maximized by stripes with $\phi=0.5$.
\end{abstract}

\section{INTRODUCTION}

Superhydrophobic (Cassie) surfaces are able to trap air at the liquid-solid interface, leading to remarkable wetting properties, such as a very large water contact angles and low hysteresis [1]. They can also have an impact on the dynamics of the liquid. For instance, the water drop slides or rolls with amazingly large velocity, and a drop hitting such a material just bounces off [2]. These macroscopic dynamic studies raised a question of a remarkable drag reducing ability of superhydrophobic materials, which could be extremely important for microfluidic lab-on-a-chip systems [ 3 [ [6] . The flow of liquids near superhydrophobic surfaces is a subject that currently attracts much experimental [7 9], simulation [10-12], and theoretical 13 18 research efforts.

Such a superlubrication potential could also dramati- cally modify a hydrodynamic interaction between different surfaces. Therefore, it is attractive to consider the hydrodynamic interaction of a hydrophilic sphere with a superhydrophobic surface. Such a configuration is relevant for many surface forces apparatus and atomic force microscope dynamic force experiments [19, 20], it represents a typical situation of phenomena of "viscous adhesion", coagulation, and more. However, despite its importance for force experiments and numerous applications, the quantitative understanding of the problem is still challenging.

The exact solution, valid for an arbitrary separation, for the drag force on a sphere moving towards a flat wall is known only for a situation when both interacting surfaces are hydrophilic (i.e. characterized by hydrodynamic no-slip boundary conditions). This was derived by Brenner [21] and Maude 22] and is given by

$$
\begin{aligned}
& F_{B M}=-\frac{1}{3} F_{S t} \sinh \xi\left(\sum_{n=1}^{\infty} \frac{n(n+1)\left[8 e^{(2 n+1) \xi}+2(2 n+3)(2 n-1)\right]}{(2 n-1)(2 n+3)\left[4 \sinh ^{2}\left(n+\frac{1}{2}\right) \xi-(2 n+1)^{2} \sinh ^{2} \xi\right]}\right. \\
& \left.-\sum_{n=1}^{\infty} \frac{n(n+1)\left[(2 n+1)(2 n-1) e^{2 \xi}-(2 n+1)(2 n+3) e^{-2 \xi}\right]}{(2 n-1)(2 n+3)\left[4 \sinh ^{2}\left(n+\frac{1}{2}\right) \xi-(2 n+1)^{2} \sinh ^{2} \xi\right]}\right),
\end{aligned}
$$


Here, $F_{S t}$ is the Stokes drag on a sphere moving in an unbounded fluid,

$$
F_{S t}=6 \pi \mu v R,
$$

$\mu$ is the dynamic viscosity of the liquid, $v$ and $R$ are the velocity and the radius of the sphere, $\cosh \xi=h / R$, $\xi<0$, and $h$ is the distance between the apex of the sphere and the wall.

Let us remark that at large separations, $h \gg R$, the two-term expansion of Eq. (11) gives 22]

$$
F_{M} \simeq F_{S t}\left(1+\frac{9}{8} \frac{R}{h}\right) .
$$

Eq. (3) is surprisingly accurate even outside of the range of its formal applicability, and can be used in a very large interval of $h / R$. However at small distances Eq.(3) gives $F_{M} / F_{S t} \simeq 9 R /(8 h)$, and therefore deviates from the Taylor formula rigorously derived for the lubrication limit, $h / R \ll 1$ :

$$
F_{T} \simeq F_{S t} \frac{R}{h} .
$$

Eqs.(1) and (4) have been verified for hydrophilic surfaces 23 26]. However, their use in other situations is beset with difficulties. There is a large literature describing attempts to answer questions of a validity of Eqs. (1) and (44) for hydrophobic and heterogeneous surfaces, and to provide a more general theory of hydrodynamic interactions. We mention below what we believe are the more relevant contributions, concentrating on the case of a hydrophilic sphere and hydrophobic or superhydrophobic flat wall.

Hydrophobic surfaces reduce drag, which is associated with a partial slippage of the fluid (characterized by a constant scalar slip length) [27, 28]. Although there is some literature describing the motion of a hydrophilic sphere parallel to a hydrophobic surface [29 31], information about the motion towards a slippery wall is rather scarce. Vinogradova proposed a modification to the Taylor equation, Eq. (44) 32, 33]. She has argued that it is convenient to describe a modification of a drag force in terms of a correction for slippage:

$$
f^{*} \simeq \frac{F_{z}}{F_{T}},
$$

and suggested general analytical expressions to relate $f^{*}$ and the slip length of interacting bodies. In case of an interaction of a hydrophilic sphere with a hydrophobic plane with a slip length $b$, the theory predicts

$$
\begin{aligned}
f^{*} \simeq \frac{F}{F_{T}}=\frac{1}{4}\left(1+\frac{3 h}{2 b}[\right. & \left(1+\frac{h}{4 b}\right) \times \\
& \left.\left.\times \ln \left(1+\frac{4 b}{h}\right)-1\right]\right) .
\end{aligned}
$$

In what follows $f^{*}$ can significantly decrease the hydrodynamic resistance force provided $h$ is of the order of
$4 b$ or smaller. In case of a perfect slip, $b=\infty$, Eq.(6) predicts $f^{*}=1 / 4$. The drag force however still remains inversely dependent on the gap as it is predicted by the Taylor theory. Later Lauga and Squires studied a sphere approaching a hydrophobic wall at very large separations, and obtained analytically a kind of small, of the order of $R / h$, correction to Eq. (33) [34]. We are unaware of any previous work that has addressed the question of hydrodynamic interaction with a hydrophobic plane at arbitrary separation and sphere radius. Note however that by symmetry reasons the problem of an interaction of a sphere with a perfectly slipping wall $(b=\infty)$ is equivalent to that of the motion of two equal spheres (separated by a twice as large distance) towards each other [35, 36].

The flow past superhydrophobic surfaces is more complex. In this situation it is advantageous to construct the tensorial effective slip boundary condition, which mimics the actual one along the true heterogeneous surface [3, 12, 37, 38]. During the last few years several papers have been concerned with the interaction of a sphere with heterogeneous superhydrophobic surfaces. Very recently Asmolov et al. developed a theory in the limit $h \ll R, L \ll R$, where $L$ is a period of the texture [39]. For the general case $h=O(L)$ the force should be found numerically. However, for some limiting cases the asymptotic equations have been derived. When the gap is large compared to the texture lengthscale, $L \ll h \ll R$, the force reads [39, 40]

$$
f^{*} \simeq 1-\frac{b_{\mathrm{eff}}^{\|}+b_{\mathrm{eff}}^{\perp}}{2 h},
$$

with $b_{\text {eff }}^{\|}$longitudinal and $b_{\text {eff }}^{\perp}$ transverse slip lengths. In the opposite limiting case of a thin gap, $h \ll \min \{b, L\} \ll$ $R$, the correction for a superhydrophobic slip depends on the fraction of a gas/liquid area only [39, 41].

The purpose of this lengthy introduction has been to show that despite its importance for dynamic force and particle suspensions experiments, the hydrodynamic interactions of a hydrophilic sphere with a superhydrophobic (and even hydrophobic) plane remain poorly understood. A key difficulty is that there is no general analytical or semi-analytical theory describing hydrodynamic flows even on smooth hydrophobic surfaces, and on composite surfaces analytical results (and in fact even numerical results) for the drag force have only been obtained in simple lubrication geometries and specific limits. Therefore, many aspects of a hydrodynamic interaction of a sphere with superhydrophobic surfaces have been given insufficient attention. The present paper employs the lattice Boltzmann simulation to extend and generalize earlier analysis.

Our paper is arranged as follows. In Sec.II we define our system, summarize earlier relevant hydrodynamic relationships and present some new theoretical estimates. Sec.III discusses our simulation method and justifies the choice of parameters. Results are discussed in Sec.IV and we conclude in Sec.V. 


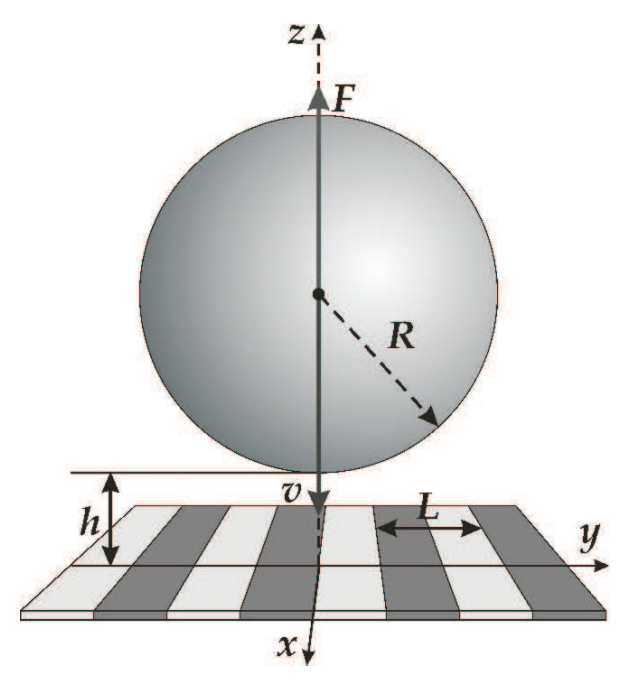

FIG. 1: Sketch of the simulated system. A sphere is approached towards a striped superhydrophobic wall.

\section{MODEL AND THEORETICAL ESTIMATES}

We first present our model and basic theoretical relationship relevant for our analysis. Here, we mostly make use of the results presented earlier with some new interpretation.

We consider a hydrodynamic interaction of a hydrophilic sphere with an idealized superhydrophobic surface in the Cassie state (sketched in Fig. 1), where a liquid slab lies on top of the surface roughness. To illustrate the approach, we focus here only on a periodically striped surface. Such canonical textures are often employed in experiments and are convenient to explore the basic physics of the system since the local (scalar) slip length varies only in one direction and is piecewise constant, thus allowing us to highlight effects of anisotropy.

The liquid/gas interface is assumed to be flat with no meniscus curvature, so that the modeled superhydrophobic surface appears as a perfectly smooth with a pattern of boundary conditions. Similar assumptions have been made in most previous publications [13, 16, 41, 42]. In this idealization, we have neglected an additional mechanism for a dissipation connected with the meniscus curvature [13, 43, 44], which may have an influence on a hydrodynamic force. Note however, that such a situation is not unrealistic, and has been achieved in many recent experiments [8, 19, 45].

Let $\mathbf{u}_{\tau}=\left(u_{x}, u_{y}, 0\right)$ be the fluid velocity along the wall, and $b(y)$ is the local slip length, which switches between two values, $b=\infty$ over gas/liquid regions and $b=0$ over solid/liquid areas. This means that we set the shear-free boundary condition over the gas/liquid regions,

$$
\frac{\partial \mathbf{u}_{\tau}}{\partial z}=\mathbf{0}
$$

and the no-slip boundary condition at a solid/liquid in- terface,

$$
\mathbf{u}_{\tau}=\mathbf{0}
$$

We remark that by assuming (8), the viscous dissipations in the gas phase, which are expected to decrease the local slip length according to a "gas cushion model" [32], have been neglected. Therefore our present results thus propose an upper bound for the local slip length at the gas areas. They could be generalized to the situation with a finite (i.e. of the order of $L$ and smaller) slip length $b(y)$ on the gas/liquid interface, but we leave this generalization for future work. By assuming no-slip, Eq. (9), at the solid area we neglect a hydrophobic slippage, which is justified provided the nanometric slip length at solid areas is small compared to a texture period [24, 46 48].

The fraction of gas/liquid area is given by $\phi$, and the fraction of solid/liquid areas is then $1-\phi$. When $\phi=0$ and $\phi=1$ the wall becomes homogeneous.

In the limit of a thick channel $(h \gg L)$ the eigenvalues of the slip-length tensor are given by [49]

$$
b_{\mathrm{eff}}^{\perp} \simeq \frac{L}{2 \pi} \ln \left[\sec \left(\frac{\pi \phi}{2}\right)\right], \quad b_{\mathrm{eff}}^{\|} \simeq 2 b_{\mathrm{eff}}^{\perp} .
$$

As proven in 50], Eq. (10) is also accurate and can safely be used in case of partial slip at the gas sectors, provided $b \gg L$. The correction to Eq. (4) is then

$$
f^{*} \simeq 1-\frac{3 L}{2 h} \ln \left[\sec \left(\frac{\pi \phi}{2}\right)\right] .
$$

In the limit of a thin channel $(h \ll L)$ with one hydrophilic and one superhydrophobic wall the effective slip of a surface decorated by perfectly slipping stripes is [15]

$$
b_{\mathrm{eff}}^{\perp} \simeq h \frac{(1-\phi)}{4 \phi}, \quad b_{\mathrm{eff}}^{\|} \simeq 4 b_{\mathrm{eff}}^{\perp}
$$

and the correction for superhydrophobic slip takes the form 39, 41.

$$
f^{*} \simeq \frac{2(4-3 \phi)}{8+9 \phi-9 \phi^{2}}
$$

\section{SIMULATION}

In this section we present our simulation method and justify the choice of parameters.

For our computer experiment we chose a scheme based on the lattice Boltzmann method which was successfully employed earlier in comparable contexts [12, 23, 51, 52]. Here, the lattice Boltzmann method serves as a NavierStokes solver for the bulk fluid flow [53]. Moving boundaries are employed to describe the momentum exchange between the fluid and a suspended sphere following the method summarized in Ref. [54]. In addition, an on-site slip boundary condition is used to describe the striped 
substrate [55, 56]. A more detailed introduction to the simulation algorithm follows below.

The Boltzmann equation

$$
\left[\frac{\partial}{\partial t}+\mathbf{u} \cdot \nabla_{\mathbf{r}}\right] f(\mathbf{r}, \mathbf{u}, t)=\Omega
$$

expresses the dynamics of the single particle probability density $f(\mathbf{r}, \mathbf{u}, t)$, where $\mathbf{r}$ is the position, $\mathbf{u}$ is the velocity, and $t$ is the time. The left-hand side of Eq. (14) models the propagation of particles in phase space, while the right hand side accounts for particle interactions by means of the collision operator $\Omega$.

By discretizing positions, velocities and time, a discrete variant of Eq. (14) can be obtained which is known as the lattice-Boltzmann equation

$$
f_{k}\left(\mathbf{r}+\mathbf{c}_{k}, t+\Delta t\right)-f_{k}(\mathbf{r}, t)=\Omega_{k}, \quad k=0,1, \ldots, B .
$$

The lattice-Boltzmann equation describes the kinetics in discrete time- $(\Delta t)$ and space-units $(\Delta x)$. In the scope of this work we employ the so-called D3Q19 lattice, referring to 3 dimensions and 19 discrete velocity vectors $\mathbf{c}_{k} ; k=\{0 . .18\}$ in direction of the $B=18$ nearest neighbors of a cube as well as a zero velocity. For convenience and without loss of generality, the scaling factors $\Delta x$ and $\Delta t$ are chosen to be of unity throughout the remainder of the text.

The use of the lattice Bhatnagar-Gross-Krook collision operator

$$
\Omega_{k}=-\frac{1}{\tau}\left(f_{k}(\mathbf{r}, t)-f_{k}^{e q}(\mathbf{u}(\mathbf{r}, t), \rho(\mathbf{r}, t))\right),
$$

which assumes relaxation on a linear timescale $\tau$ towards a discretized local Maxwell-Boltzmann distribution $f_{k}^{e q}$, is sufficient to recover a second order accurate solution of the Navier-Stokes equations [57]. The kinematic viscosity $\nu=(2 \tau-1) / 6$ of the fluid is related to the relaxation time scale. In this study the latter is kept constant at $\tau=1$. Macroscopic flow properties can be related to stochastic moments of $f$, where the fluid density $\rho(\mathbf{r}, t)=\rho_{0} \sum_{k} f_{k}(\mathbf{r}, t)$ and momentum $\rho(\mathbf{r}, t) \mathbf{u}(\mathbf{r}, t)=\rho_{0} \sum_{k} \mathbf{c}_{k} f_{k}(\mathbf{r}, t)$ are of special interest $\left(\rho_{0}\right.$ denotes a reference density).

We use a simulation cell confined by two impermeable walls at $z=N_{z}$ and $z=0$. At an upper wall we apply noslip boundary conditions by means of a simple mid-grid bounce back boundary condition. A bottom wall is modeled by patterns of a local slip length on a planar surface. More specifically, we impose patches of zero and infinite slip-lengths by using (a second order accurate) fixed velocity boundary condition as it was described in [56] and then used in several applications in [12, 52, 55]. The alternating perfect slip and no-slip stripes are aligned in $y$-direction and are of the widths $\phi L$ and $(1-\phi) L$, respectively. We vary $\phi$ with the step size of 0.25 from 0 to 1. In $x$ - and $y$-directions the simulation domain is limited by periodic boundaries.
Following the approach of Ladd and coworkers [54], the hydrophilic sphere is implemented as a no-slip boundary moving with constant velocity $\mathbf{v}$. To determine the momentum transferred to the fluid as the sphere moves, both the center of mass velocity and the particle rotation are taken into account. While the boundary moves on the fluid lattice, its discretization is constantly adopted. This causes fluctuations in the measured forces which we suppress by averaging the measurement over approximately 1000 timesteps [23, 51]. The system resolution has to be balanced between an optimal approximation of the model and computational cost.

The cell height $N_{z}$ has to be sufficiently large to allow to disregard the influence of the upper wall on the flow field. However, it should be as small as possible to keep calculation times within acceptable limits. To find the most suitable $N_{z}$, we studied the influence of the upper surface on the results. When the sphere radius is small compared to the height of the box, the first-order correction to the drag due to the upper wall is inversely proportional to its separation from the sphere [35]. We thus propose the following equation to fit the data:

$$
F_{z}=F_{\text {theor }}+F_{S t} \frac{R}{N_{z}-2 R-h},
$$

where $F_{\text {theor }}$ is the theoretical prediction for the drag in the absence of the upper wall. Eq. (17) allows us to estimate the effect of the upper wall on the interaction for different $N_{z}$. We simulated the drag force acting on a sphere approaching the homogeneous bottom wall, and considered two limiting cases. Namely, of a no-slip bottom wall, $\phi=0$, and of a perfectly slipping wall, $\phi=1$, where exact theoretical solutions are known. We recall that in case of a no-slip wall the drag force is given by Eq.(11), and that in case of perfectly slipping wall the drag force is equivalent to that for two approaching spheres at twice larger distance [35, 36]. We have plotted simulation results obtained with $N_{z} / R=8$ and $N_{z} / R=16$ in Fig. 2, Also included are the theoretical predictions and the theoretical values recalculated by using Eq. (17). A general conclusion from this plot is that $N_{z} / R=16$ allows us to neglect the influence of the upper wall for $h / R<1$. For smaller cell height, $N_{z} / R \leq 16$, or larger gaps, $h / R \geq 1$, the influence of the upper boundary cannot be ignored. Note however that the data for $N_{z} / R=8$ are in good agreement with predictions of Eq. (17).

Similar remarks concern the lateral size of the system, which should be sufficiently large to minimize (without exceeding feasible computation times) the influence of mirror spheres acting across the periodic boundaries. The periodic boundary conditions can be properly accounted for by introducing an effective corrected radius, which difference from $R$ does not exceed $2 \%$ for $N_{x} / R=N_{y} / R=8$ [23, 51]. Finally, we note that the radius of the sphere should also be large enough to model analogy with an actual sphere rather than a staircased construction. A further requirement introduced by the theory is the radius to be significantly larger than $L$. 


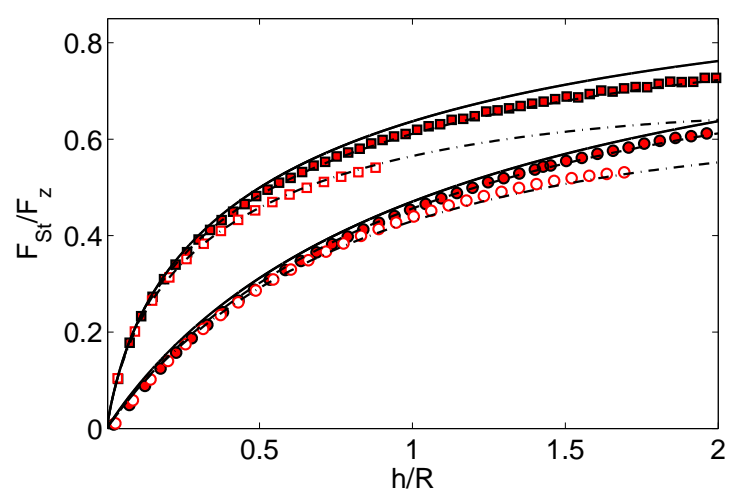

FIG. 2: The drag force vs. separation curve obtained for two different heights of the simulation cell, $N_{z} / R=8$ (open symbols) and $N_{z} / R=16$ (red symbols). The upper data set corresponds to the interaction of a sphere with the no-slip wall. The lower data set is obtained for the interaction with the perfectly slipping wall. Solid lines show the theoretical value calculated using Eq.(11). Dashed-dotted lines are given by Eq. (17).

However, the width of a single stripe has to be resolved by at least four lattice sites to avoid unacceptable discretization errors [23, 51, 55].

Taking into account above constraints and comparing different possible configurations, we have determined a minimal set of simulation parameters given by: $N_{x}=$ $N_{y}=256, N_{z}=512, R=32, L=16$. Furthermore, selected runs have been repeated at double resolution in order to approximate the discretization errors introduced.

\section{RESULTS AND DISCUSSION}

In this Section we present our simulation results. More specifically, we discuss simulation data obtained for normal and lateral forces in the system, and also give some detailed analysis of a fluid velocity field and a pressure distribution.

\section{A. Normal force}

Here we present the simulation results for the interaction of a sphere with striped surfaces defined by different fractions of the gas phase. In order to assess the validity of the above lattice-Boltzmann approach, we first measure the hydrodynamic force on a sphere approaching uniform non-slipping $(\phi=0)$ and perfectly $(\phi=1)$ smooth walls, where theoretical solutions are known, i.e. verify Eq.(11) and results of [35, 36]. Fig. 3(a) shows the simulation results and theoretical curves for a normalized vertical force, $F_{z} / F_{S t}$, as a function of $h / R$. The quantitative agreement between the simulation and theoretical
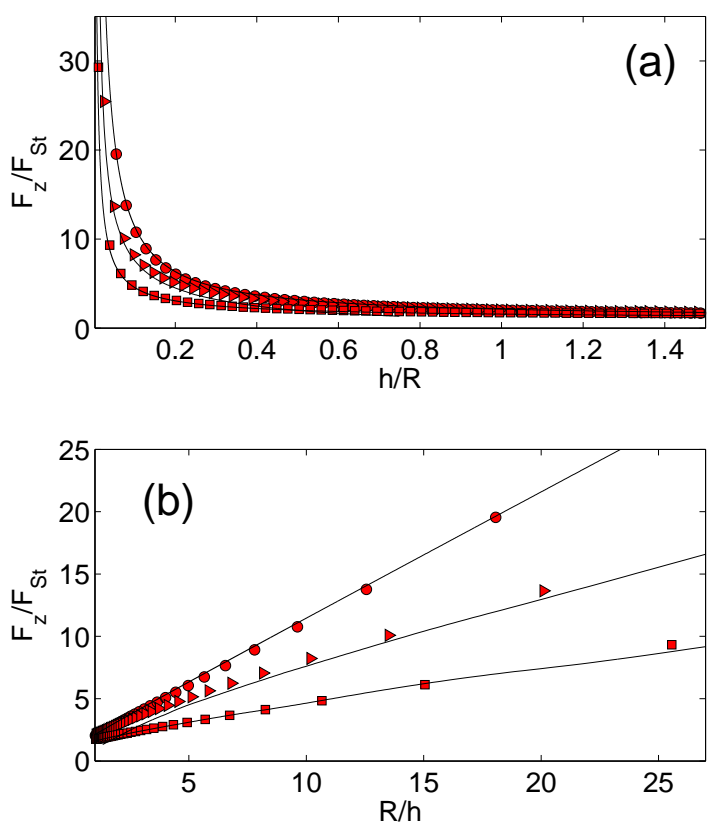

FIG. 3: Hydrodynamic force on a sphere approaching a wall plotted in different coordinates. Symbols from top to bottom are the simulation data for $\phi=0,0.5$ and 1 . Solid curves show theoretical predictions.

results is excellent for all separations. This demonstrates the accuracy of our simulations. It can be seen that in case of a perfectly slipping wall the force at small distances is much smaller than predicted by Eq.(11). To examine the short-distance region in more detail the data from Fig. 3(a) are reproduced in Fig. 3(b) in different coordinates. Fig. 3(b) is intended to indicate that shortdistance theoretical asymptotics for smooth surfaces is well reproduced in simulations, and we emphasize that it is well seen that in both cases when $h \ll R$ the force becomes inversely proportional to the gap as predicted by Eqs.(4) and (6). It is intuitively clear that in case of patterned superhydrophobic surfaces the force should be confined between these two limiting curves. The force curve simulated with $\phi=0.5$ is included in Fig. 3. Note that here and in simulations below the default location of the apex of the sphere is above the boundary between no-slip and perfect-slip stripes (as shown in Fig. 1) unless another configuration is specified. The computed curve indeed shows the drag smaller than for a no-slip wall, but larger than for a perfectly slipping wall. Fig. 3 includes theoretical curves calculated within the lubrication approach (i.e. justified provided $h \ll R$ ) [39]. A striking result is that predictions of the lubrication-type theory are in a very good quantitative agreement with simulation results.

We now investigate the effect of the gas fraction, $\phi$, on the drag force. The measured data are presented in 


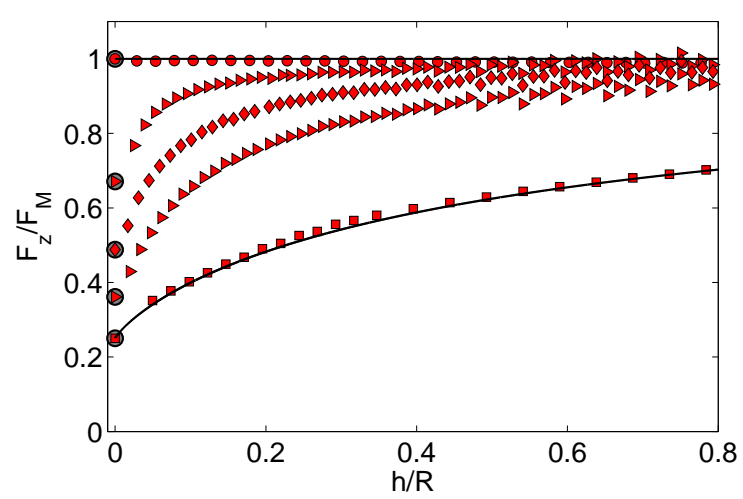

FIG. 4: Correction for superhydrophobic slippage, $F_{z} / F_{M}$ vs. $h / R$ (red symbols). From top to bottom $\phi=0,0.25,0.5,0.75$, and 1. Grey circles show the predictions of Eq. (13). Solid curves plot the theoretical results for no-slip and perfectly slipping walls.

Fig. 4, which shows $F_{z} / F_{M}$ as a function of $h / R$. The ratio $F_{z} / F_{M}$ denotes the correction for superhydrophobic slippage to the force expected between two hydrophilic surfaces. This is well seen in Fig. 4, which indicates that $F_{z} / F_{M}=1$ at $\phi=0$. It can also be seen that the correction factor decreases with $\phi$, i.e. the force becomes smaller than predicted by Eq.(11). As expected, all curves are confined between two limiting forces, obtained for $\phi=0$ and $\phi=1$. We should like to stress that the correction for superhydrophobic slippage is long-range, and the deviations from Eq.(1) are discernible even at $h / R=O(1)$, especially for large $\phi$. At small $h / R$ this discrepancy becomes significant, and $F_{z} / F_{M}$ approaches its minimal value at a given $\phi$. In the lubrication limit, $R \gg L \gg h$, these values can be evaluated with Eq.(13). Given the approximations required to derive Eq.(13) we do not expect it to be accurate for our system since the sphere radius in the simulation is not large enough, $R / L=2$. However, calculations with Eq.(13) shown in Fig. 4 coincide with the simulation. This surprising result suggests that Eq.(13) is more general that it was assumed originally.

\section{B. Fluid velocity and pressure distribution}

Since our wall is a highly anisotropic striped surface it is instructive to study the velocity and excess (as compared to the ambient pressure at infinity) pressure, $p$, in the gap.

Fig. 5 shows vector $\mathbf{u}_{\tau}$ and contour $\left|\mathbf{u}_{\tau}\right|$ fields measured for $h=3 L / 4$ in the cross-section close to the wall, $z=L / 8$. We see that the velocity is nonuniform throughout the liquid. Note that there is a discernible asymmetry of the flow around the $x$-axis, which obviously reflects the location of the sphere apex above the edge of the stripes. The velocity contour lines are significantly elon-

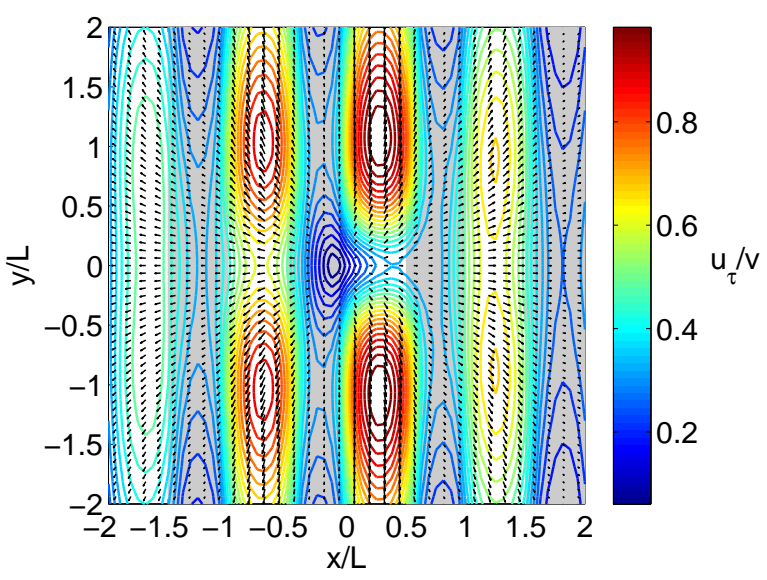

FIG. 5: Vector and contour fields of the lateral velocity at the cross-section $z=L / 8$ simulated for $h=3 L / 4$. No-slip stripes are shown by grey, and perfect-slip stripes by white color.

gated in the longitudinal direction, which indicates that liquid preferably flows along the stripes. The velocity is very small near the center, $x=y=0$, and at large distances from the axis, $x^{2}+y^{2} \gg L^{2}$, where the gap becomes large. It has been earlier predicted that for noslip 25] and uniformly slipping [58] walls, where the flow is radially symmetric, and at thin gaps, $h \ll R$, the maximum velocity is attained at distances from the center of the order of $(R h)^{1 / 2}$. It can be seen that the maximum velocity in our case is also observed at distances of the order of $(R h)^{1 / 2}$. However, due to a strong anisotropy of the flow we also observe local velocity maxima and minima over perfect-slip (white regions) and no-slip stripes (grey regions), respectively.

To examine the significance of anisotropy of the texture in more details, we have investigated the distributions of lateral velocity and the excess pressure in the longitudinal and transverse directions. Figs. 6(a) and (b) show the velocity in eigendirections measured at two cross-sections. One is chosen to be in the vicinity of the wall $\left(z_{1}=L / 8\right)$, and another one is closer to the sphere $\left(z_{2}=L / 2\right)$. It can be seen that at a small distance from the striped wall (Fig. 6(a)), the longitudinal velocity behaves qualitatively similar to what is expected for uniform walls [25, [58]. More precisely, it is zero near the origin of coordinates, has maxima at some distance from it, and decays at larger distances. The transverse velocity shows irregular oscillatory behavior, which is a reflection of the striped texture since the oscillations have a period $L$. The oscillation maxima nearly coincide with the amplitude of the longitudinal velocity and are detected at the middle of the perfect-slip stripes. The minima are observed at the centers of no-slip regions, and here the transverse velocity is much smaller than longitudinal. This result is similar to obtained earlier for weaklyslipping stripes [52]. At larger distance from the striped wall (Figs. 6(b)), the flow is nearly isotropic, and we do 

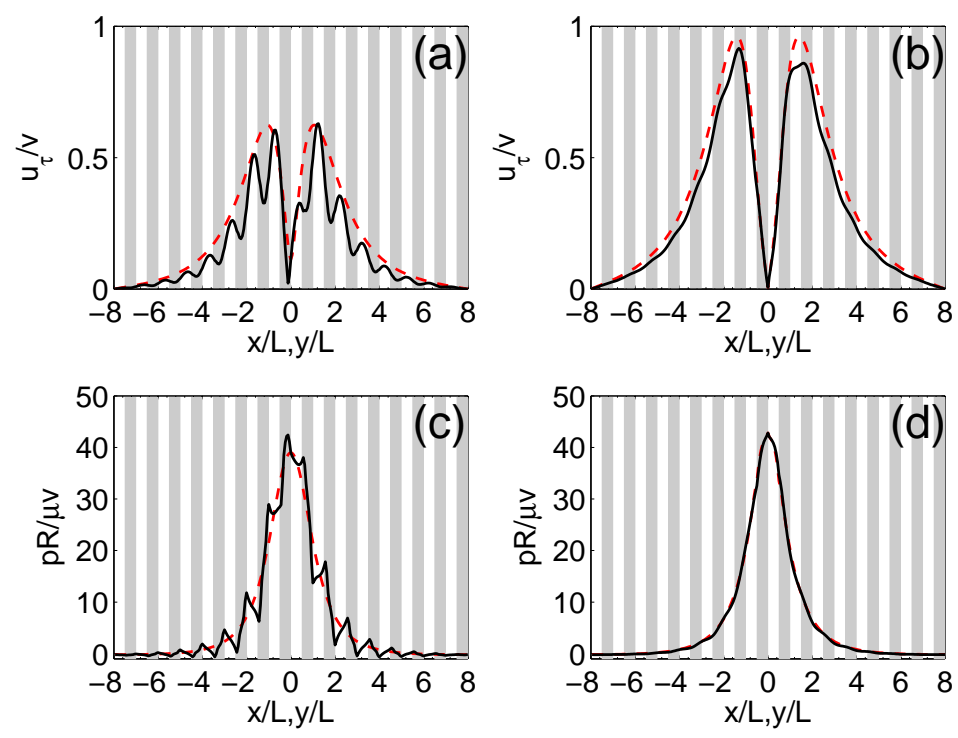

FIG. 6: Distributions of lateral liquid velocity and pressure taken at the cross-sections $z=L / 8$ (a, c) and $z=L / 2$ (b, d) and in longitudinal (dashed curves) and transverse (solid curves) directions. Measurements are performed at the fixed gap thickness $h=3 L / 4$. No-slip and perfect-slip regions are shown by gray and white stripes.

not observe any oscillation of the amplitude of the transverse velocity. We also note that this cross-section is characterized by a faster flow.

In Figs. 6(c) and (d) we plot the simulated pressure at the same cross-sections. Perhaps the most important conclusion from these plots is that despite an anisotropy of the flow, the averaged over the texture period $L$ pressure is nearly isotropic. Similar results have been already obtained numerically, but only in the lubrication limit, $L, h \ll R[39$. Another important point to note is that pressure is the same for the two cross-sections, i.e. it does not depend on $z$ being nearly constant across the gap, as in the lubrication theory. Abrupt changes of the pressure in the transverse direction can be seen in Fig. 6(c), which corresponds to the cross-section close to the wall. In contrast to oscillations of a transverse velocity, the local maxima and minima of pressure are located at the boundary between perfect-slip and no-slip stripes. The pressure for flows across the stripes was predicted to diverge at the wall, $z=0$, near the jump in $b(y)$ : $p \propto r^{-1 / 2}$, where $r \ll L$ is the distance from the border between stripes [16]. Our simulation results do confirm qualitatively these theoretical predictions, but of course a quantitative agreement cannot be obtained since we measure pressure at some finite distance from the wall, and also because in simulations pressure is always finite.

\section{Lateral force}

Finally, we consider the lateral force on the sphere, which could be present in addition to the normal drag force. In most real experiments the alignment of the sphere and stripes is inconvenient or difficult, so the sphere could be in different positions relative to the wall texture. In our simulations we explore four representative cases as shown in Fig. 7 (a). Two configurations, 1 and 3, where the apex of the sphere is located above the center of a no-slip or a perfect slip stripe, are symmetric. Therefore, the lateral force is expected to be absent. Since the asymmetry is maximized in two other configurations, 2 and 4, where the sphere is above the border between stripes, we consider them with the goal to maximize the lateral force. We remark that our above analysis corresponds to a sphere in a position 2 .

We show in Fig. 7 (b) the simulation results for the lateral force obtained with $\phi=0.5$, which corresponds to maximum transverse flow in a thin channel situation [11, 59]. The simulation data demonstrates that the lateral force is measurable only when the sphere is very close to the wall, $h / R \leq 0.05$, and that it is three orders of magnitude smaller than the normal drag force. These results also clearly show the importance of a location of a sphere relative to stripes in generating the lateral force. They illustrate that the lateral force vanishes in positions 1 and 3 , is positive for position 2 , and negative for position 4 . This indicates that in the asymmetric position the lateral force pushes the sphere towards the center of the perfect-slip stripes, where the friction is lower. We remark that recently Pimponi et al. studied numerically the particle motion in the vicinity of the striped wall and reported similar behavior of a lateral force of small magnitude [60].

The detailed comparison between the simulation re- 

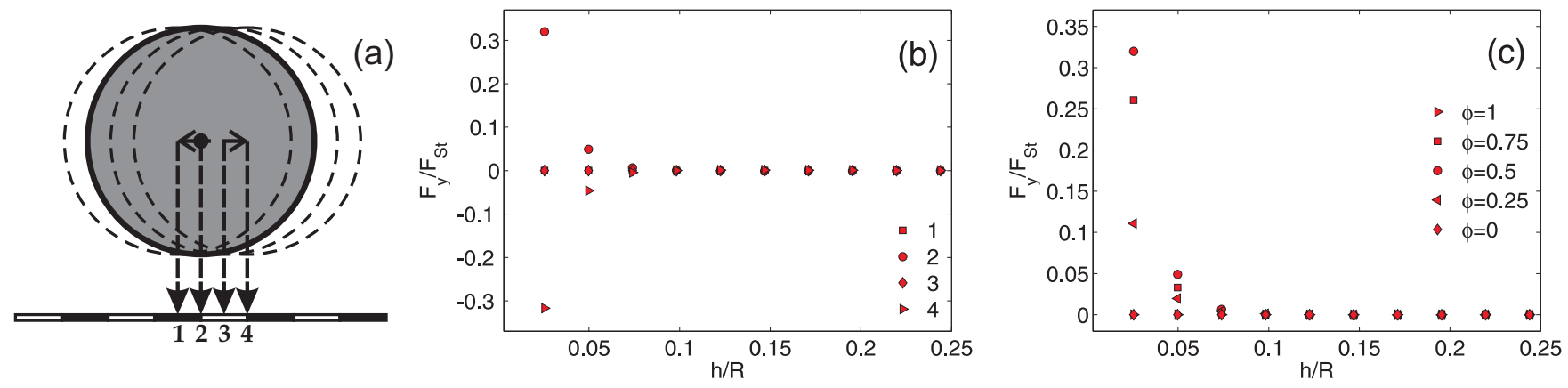

FIG. 7: (a) Illustration of four possible sphere positions relative to the texture. (b) Lateral forces at fixed $\phi=0.5$ simulated for different locations of the sphere relative to stripes. (c) Lateral forces for a sphere in position 2 simulated with different $\phi$.

sults obtained with a different fraction of the gas/liquid area is then shown in Fig. 7(c). We see that the maximal force is indeed obtained for the stripes with equal area fractions, $\phi=0.5$, which correlates well with earlier theoretical predictions of transverse phenomena in thin channels [59]. The weaker lateral force is detected for $\phi=0.25$ and 0.75 , and it disappears for a homogeneous surface, $\phi=0$ and 1 .

Finally, coming back to the normal force, a similar study of the role of the sphere location show that the changes in the normal force are detectable at $h \ll R$, but they never exceeds $1 \%$ and can therefore safely be ignored. As a side note, one may remark that all our results have been obtained for $R=O(L)$ and could not be immediately applied to the situation of $R \ll L$, where the effect of a sphere location on the normal force might become significant. This case however is beyond the scope of present work and will be discussed elsewhere.

\section{CONCLUSION}

In conclusion, we have presented simulation data for a hydrodynamic interaction of a sphere moving towards a superhydrophobic striped plane. We checked the validity of our approach by reproducing the known theoretical predictions for uniform no-slip and perfectly slipping walls. The simulation results show that the drag force acting on a sphere approaching the striped wall is confined between these two limiting solutions, and that the magnitude of the force depends strongly on the fraction of gas/liquid areas. A quantitative agreement with earlier predictions of a force in the limit of a thin gap has been obtained. We have also examined the flow field and detected an oscillatory character of its transverse component in the vicinity of the wall, which reflects the influence of the heterogeneity and anisotropy of the striped texture. Our analysis of pressure data led to a conclusion that despite an anisotropy of the texture the average pressure in the gap remain surprisingly isotropic. However, in the vicinity of the wall we observed abrupt jumps in pressure in the transverse direction, which correlate well with earlier predicted singularities at the border of no-slip and perfect-slip stripes. Finally, we investigated the lateral force on the sphere, and found that it is detectable in the case of the thin gap and also depends strongly on the fraction of the gas areas.

\section{ACKNOWLEDGEMENT}

This research was partly supported by the Russian Academy of Science (RAS) through its priority program 'Assembly and Investigation of Macromolecular Structures of New Generations' (grant of O. I. Vinogradova), and by the Netherlands Organization for Scientific Research (NWO/STW VIDI grant of J. Harting and NWO travel grant of E.S. Asmolov). We acknowledge computing resources from the Jülich Supercomputing Center, the Scientific Supercomputing Center Karlsruhe and SARA Amsterdam.
[1] D. Quere, Rep. Prog. Phys. 68, 2495 (2005).

[2] P. Tsai, R. C. A. van der Veen, M. van de Raa, and D. Lohse, Langmuir 26, 16090 (2010).

[3] O. I. Vinogradova and A. V. Belyaev, J. Phys.: Condens. Matter 23, 184104 (2011).

[4] J. P. Rothstein, Annu. Rev. Fluid Mech. 42, 89 (2010).
[5] O. I. Vinogradova and A. L. Dubov, Mendeleev Commun. 22, 229 (2012).

[6] G. McHale, M. I. Newton, and N. J. Schirtcliffe, Soft Matter 6, 714 (2010).

[7] C.-H. Choi, U. Ulmanella, J. Kim, C.-M. Ho, and C.-J. Kim, Phys. Fluids 18, 087105 (2006). 
[8] E. Karatay, A. S. Haase, C. W. Visser, C. Sun, D. Lohse, P. A. Tsai, and R. G. H. Lammertink, PNAS 110, 8422 (2013).

[9] P. Joseph, C. Cottin-Bizonne, J. M. Benoř, C. Ybert, C. Journet, P. Tabeling, and L. Bocquet, Phys. Rev. Lett. 97, 156104 (2006).

[10] N. V. Priezjev, J. Chem. Phys. 135, 204704 (2011).

[11] J. Zhou, A. V. Belyaev, F. Schmid, and O. I. Vinogradova, J. Chem. Phys. 136, 194706 (2012).

[12] S. Schmieschek, A. V. Belyaev, J. Harting, and O. I. Vinogradova, Phys. Rev. E 85, 016324 (2012).

[13] C. Ybert, C. Barentin, C. Cottin-Bizonne, P. Joseph, and L. Bocquet, Phys. Fluids 19, 123601 (2007).

[14] C. Cottin-Bizonne, C. Barentin, E. Charlaix, L. Bocquet, and J.-L. Barrat, Eur. Phys. J. E 15, 427 (2004).

[15] F. Feuillebois, M. Z. Bazant, and O. I. Vinogradova, Phys. Rev. Lett. 102, 026001 (2009).

[16] E. S. Asmolov and O. I. Vinogradova, J. Fluid Mech. 706, 108 (2012).

[17] C. Cottin-Bizonne, C. Barentin, and L. Bocquet, Phys. Fluids 24, 012001 (2012).

[18] D. Crowdy, Phys. Fluids 22, 121703 (2010).

[19] A. Steinberger, C. Cottin-Bizonne, P. Kleimann, and E. Charlaix, Nature Materials 6, 665 (2007).

[20] A. Maali and B. Bhushan, Phil. Trans. R. Soc. A 370, 2304 (2012).

[21] H. Brenner, Chem. Eng. Sci. 16, 242 (1961).

[22] A. D. Maude, Brit. J. Appl. Phys. 12, 293 (1961).

[23] C. Kunert, J. Harting, and O. Vinogradova, Phys. Rev. Lett. 105, 016001 (2010).

[24] O. I. Vinogradova and G. E. Yakubov, Langmuir 19, 1227 (2003).

[25] D. Y. C. Chan and R. G. Horn, J. Chem. Phys. 83, 5311 (1985).

[26] C. D. F. Honing and W. A. Ducker, Phys. Rev. Lett. 98, 028305 (2007).

[27] O. I. Vinogradova, Int. J. Miner. Proc. 56, 31 (1999).

[28] J.-L. Barrat and L. Bocquet, Faraday Discuss. 112, 119 (1999).

[29] A. M. J. Davis, M. T. Kezirian, and H. Brenner, J. Colloid Interface Sci. 165, 129 (1994).

[30] H. Luo and C. Pozrikidis, J. Eng. Math. 62, 1 (2008).

[31] F. Feuillebois, N. Ghalya, A. Sellier, and L. Elasmi, Journal of Physics: Conference Series 392, 012012 (2012).

[32] O. I. Vinogradova, Langmuir 11, 2213 (1995).

[33] O. I. Vinogradova, Langmuir 12, 5963 (1996).

[34] E. Lauga and T. M. Squires, Physics of Fluids 17, 103102 (2005).

[35] J. Happel and H. Brenner, Low Reynolds number hydrodynamics: with special applications to particulate media (Martinus Nijhoff Publishers, The Hague, 1983).
[36] D. J. Jeffrey and Y. Onishi, J. Fluid Mech. 139, 261 (1984).

[37] M. Z. Bazant and O. I. Vinogradova, J. Fluid Mech. 613, 125 (2008).

[38] K. Kamrin, M. Bazant, and H. A. Stone, J. Fluid Mech. 658, 409 (2010).

[39] E. S. Asmolov, A. V. Belyaev, and O. I. Vinogradova, Phys. Rev. E 84, 026330 (2011).

[40] N. Lecoq, R. Anthore, B. Cichocki, P. Szymczak, and F. Feuillebois, J. Fluid Mech. 513, 247 (2004).

[41] A. V. Belyaev and O. I. Vinogradova, Soft Matter 6, 4563 (2010).

[42] N. V. Priezjev, A. A. Darhuber, and S. M. Troian, Phys. Rev. E 71, 041608 (2005).

[43] J. Hyväluoma and J. Harting, Phys. Rev. Lett. 100, 246001 (2008).

[44] M. Sbragaglia and A. Prosperetti, Phys. Fluids 19, 043603 (2007).

[45] A. S. Haase, E. Karatay, P. A. Tsai, and R. G. H. Lammertink, Soft Matter 9, 8949 (2013).

[46] O. I. Vinogradova, K. Koynov, A. Best, and F. Feuillebois, Phys. Rev. Lett. 102, 118302 (2009).

[47] N. V. Churaev, V. D. Sobolev, and A. N. Somov, J. Colloid Interface Sci. 97, 574 (1984).

[48] L. Joly, C. Ybert, and L. Bocquet, Phys. Rev. Lett. 96, 046101 (2006).

[49] E. Lauga and H. A. Stone, J. Fluid Mech. 489, 55 (2003).

[50] A. V. Belyaev and O. I. Vinogradova, J. Fluid Mech. 652, 489 (2010).

[51] C. Kunert and J. Harting, IMA J. Apl. Math. 76, 761 (2011).

[52] E. S. Asmolov, S. Schmieschek, J. Harting, and O. I. Vinogradova, Phys. Rev. E 87, 023005 (2013).

[53] R. Benzi, S. Succi, and M. Vergassola, Phys. Rep. 222, 145 (1992).

[54] A. J. C. Ladd and R. Verberg, J. Stat. Phys. 104, 1191 (2001).

[55] N. K. Ahmed and M. Hecht, J. Stat. Mech. 2009, 9017 (2009).

[56] M. Hecht and J. Harting, J. Stat. Mech. 2010, 1018 (2010).

[57] P. L. Bhatnagar, E. P. Gross, and M. Krook, Phys. Rev. 94, 511 (1954).

[58] R. G. Horn, O. I. Vinogradova, M. E. Mackay, and N. Phan-Thien, J. Chem. Phys. 112, 6424 (2000).

[59] F. Feuillebois, M. Z. Bazant, and O. I. Vinogradova, Phys. Rev. E 82, 055301(R) (2010).

[60] D. Pimponi, M. Chinappi, P. Gualtieri, and C. M. Casciola, Microfluidics Nanofluidics pp. DOI 10.1007/s10404013-1243-4 (2013). 